\title{
A CONVERSE OF LOTZ'S THEOREM ON UNIFORMLY CONTINUOUS SEMIGROUPS
}

\author{
J. M. A. M. VAN NEERVEN
}

(Communicated by Palle E. T. Jorgensen)

\begin{abstract}
We prove the following partial converse to a theorem of Lotz: If every $C_{0}$-semigroup on a Banach lattice $E$ with quasi-interior point is uniformly continuous, then $E$ is isomorphic to a $C(K)$-space with the Grothendieck property.
\end{abstract}

Let us say that a Banach space $X$ has the Lotz property if every $C_{0}$-semigroup on $X$ is uniformly continuous. In [L] it was shown by Lotz that every Grothendieck space with the Dunford-Pettis property has the Lotz property, after some special cases were obtained by several authors, notably Coulhon [C], KishimotoRobinson [KR], and Lotz himself. Examples of Grothendieck Dunford-Pettis spaces are $l^{\infty}$ and $L^{\infty}(\Omega, \mu)$ and, more generally, $\sigma$-Dedekind complete $C(K)$-spaces. We will give a partial converse of Lotz's result, which uses the following lemma [Ne]. A strongly continuous semigroup $\{T(t)\}_{t \geq 0}$ on a Banach lattice $E$ is called a multiplication semigroup if each $T(t)$ is a band-preserving operator.

Lemma 1. Let $E$ be a Banach lattice with quasi-interior point $u>0$. Let $v \leq 0$ be arbitrary. Then there exists a multiplication semigroup on $E$ with generator $A$ such that $u \in D(A)$ and $A u=v$.

Theorem 2. Let $E$ be a Banach lattice with quasi-interior point. Then the following assertions are equivalent

(1) E has the Lotz property,

(2) E has the Grothendieck property and the Dunford-Pettis property,

(3) $E$ is isomorphic to a $C(K)$-space with the Grothendieck property.

Proof. (1) $\Rightarrow(3)$. Let $u>0$ be a quasi-interior point of $E$. Let $v \leq 0$ be arbitrary, and let $A$ be the generator of a multiplication semigroup on $E$ with $A u=v$. Such a semigroup exists by the lemma. This semigroup is uniformly continuous by assumption, whence $A$ is a bounded band-preserving operator. By [W], such operators preserve ideals. Hence $v=A u \in E_{u}$, the ideal generated by $u$. If $v \in E$ is arbitrary, $v \in E_{u}$ follows from $v=v^{+}-v^{-}$. We have

Received by the editors March 28, 1991.

1991 Mathematics Subject Classification. Primary 47D03, 47B55, 46B30.

Key words and phrases. Strongly continuous semigroups, Grothendieck property, Dunford-Pettis property, Banach lattice with quasi-interior point. 
shown that $E=E_{u}$, in other words, $u$ is actually a strong order unit for $E$. By the Kakutani representation theorem, $E$ is Banach lattice isomorphic to a $C(K)$ space. We still have to prove that $E$ is Grothendieck. By [N] it suffices to show that $E$ does not contain a complemented subspace isomorphic to $c_{0}$. If such a subspace exists, say $E=c_{0} \oplus F$, however, then $T(t) \oplus \mathrm{id}_{F}$, where $(T(t) x)_{n}:=e^{-n t} x_{n}$, defines a strongly continuous semigroup on $E$ that is not uniformly continuous.

$(3) \Rightarrow(2)$. Every $C(K)$-space has the Dunford-Pettis property.

$(2) \Rightarrow(1)$. This follows from Lotz's theorem.

Remark. It follows from the theorem that every Grothendieck Dunford-Pettis lattice with a quasi-interior point is a $C(K)$-space.

Remark. In [Le] the so-called surjective Dunford-Pettis property is introduced. It is shown there that every Grothendiek space with the surjective DunfordPettis property has the Lotz property. Moreover, an example is constructed of a Grothendieck lattice with a weak order unit having the surjective DunfordPettis property but not the Dunford-Pettis property. This shows that Theorem 2 fails for Banach lattices with a weak order unit.

A Banach space is called weakly compactly generated (WCG) if it is the closed linear span of one of its weakly compact subsets. Every separable and every reflexive Banach space is WCG. It is well known [J] that every WCG space with the Grothendieck and the Dunford-Pettis property is finite dimensional.

Corollary 3. If an infinite-dimensional Banach lattice $E$ has the Lotz property, then $E$ cannot be weakly compactly generated.

Proof. Suppose $E$ is weakly compactly generated. If $E$ contains a copy of $c_{0}$, then by Veech's version of Sobczyk's theorem [V], this $c_{0}$ is automatically complemented. Therefore $E$ contains no copy of $c_{0}$, and hence $E$ has order continuous norm (see, e.g, [S, Theorem II.5.15]). In particular, closed ideals are projection bands. Since $E$ is infinite dimensional, there is an $u>0$ such that $\overline{E_{u}}$ is infinite dimensional. If every $C_{0}$-semigroup on $\overline{E_{u}}$ is uniformly continuous, then $\overline{E_{u}}$ is isomorphic to a $C(K)$ by Theorem 2 and hence contains a copy of $c_{0}$, a contradiction. So the complemented subspace $\overline{E_{u}}$, and hence $E$, admits a $C_{0}$-semigroup with unbounded generator.

The proof shows that a Lotz lattice cannot have order continuous norm. Hence by [S, Theorem II.5.14] we have

Corollary 4. Every infinite-dimensional $\sigma$-Dedekind complete Lotz lattice contains a sublattice isomorphic to $l^{\infty}$.

\section{ACKNOWLEDGMENT}

This paper was written during a half-year visit at the University of Tübingen. I would like to thank the CWI in Amsterdam for giving me the opportunity to visit Tübingen and all members of the faculty in Tübingen for their warm hospitality. I especially thank Frank Räbiger for drawing my attention to references [Le, N]. 


\section{REFERENCES}

[C] T. Coulhon, Suites des opérateurs sur un espace $C(K)$ de Grothendieck, C. R. Acad. Sci. Paris Sér. I Math. 298 (1984), 13-15.

[J] W. B. Johnson, No infinite-dimensional P space admits a Markusevich basis, Proc. Amer. Math. Soc. 26 (1970), 467-468.

[KR] A. Kishimoto and D. W. Robinson, Subordinate semi-groups and order properties, J. Austral. Math. Soc. Ser. A 31 (1981), 59-76.

[Le] D. Leung, Uniform convergence of operators and Grothendieck spaces with the Dunford-Pettis property, Math. Z. 197 (1988), 21-32.

[L] H. P. Lotz, Uniform convergence of operators on $L^{\infty}$ and similar spaces, Math. Z. 190 (1985), 207-220.

[Ne] J. M. A. M. van Neerven, Abstract multiplication semigroups (submitted).

[N] C. P. Niculescu, Weak compactness in Banach lattices, J. Operator Theory 6 (1981), 217 231.

[S] H. H. Schaefer, Banach lattices and positive operators, Springer-Verlag, Berlin, Heidelberg, and New York, 1974.

[V] W. A. Veech, A short proof of Sobczyk's theorem, Proc. Amer. Math. Soc. 28 (1971), 627-628.

[W] A. W. Wickstead, Representation and duality of multiplication operators on Archimedean Riesz spaces, Compositio Math. 35 (1977), 225-238.

Centre for Mathematics and Computer Science, P.O. Box 4079, 1009 AB Amsterdam, THE NETHERLANDS 